\title{
Alternative Architecture of the E. coli Chemosensory Array
}

\author{
Alister Burt ${ }^{1,+}$, C. Keith Cassidy ${ }^{2,+}$, Phillip J. Stansfeld ${ }^{3}$ and Irina Gutsche ${ }^{1, * \mathbb{C}}$ \\ 1 Institut de Biologie Structurale, Université Grenoble Alpes, CEA, CNRS, IBS, 71 Avenue des Martyrs, \\ F-38044 Grenoble, France; alister.burt@ibs.fr \\ 2 Department of Biochemistry, University of Oxford, South Parks Road, Oxford OX1 3QU, UK; \\ keith.cassidy@bioch.ox.ac.uk \\ 3 Department of Chemistry, School of Life Sciences, University of Warwick, Gibbet Hill Campus, \\ Coventry CV4 7AL, UK; phillip.stansfeld@warwick.ac.uk \\ * Correspondence: irina.gutsche@ibs.fr \\ + Equal contribution.
}

check for updates

Citation: Burt, A.; Cassidy, C.K.; Stansfeld, P.J.; Gutsche, I. Alternative Architecture of the E. coli Chemosensory Array. Biomolecules 2021, 11, 495. https://doi.org// 10.3390biom11040495

Academic Editor:

Cristina Martínez-Villaluenga

Received: 5 February 2021

Accepted: 23 March 2021

Published: 25 March 2021

Publisher's Note: MDPI stays neutral with regard to jurisdictional claims in published maps and institutional affiliations.

Copyright: (c) 2021 by the authors. Licensee MDPI, Basel, Switzerland. This article is an open access article distributed under the terms and conditions of the Creative Commons Attribution (CC BY) license (https:/ / creativecommons.org/licenses/by/ $4.0 /)$.

\begin{abstract}
Chemotactic responses in motile bacteria are the result of sophisticated signal transduction by large, highly organized arrays of sensory proteins. Despite tremendous progress in the understanding of chemosensory array structure and function, a structural basis for the heightened sensitivity of networked chemoreceptors is not yet complete. Here, we present cryo-electron tomography visualisations of native-state chemosensory arrays in E. coli minicells. Strikingly, these arrays appear to exhibit a p2-symmetric array architecture that differs markedly from the p6-symmetric architecture previously described in E. coli. Based on this data, we propose molecular models of this alternative architecture and the canonical p6-symmetric assembly. We evaluate our observations and each model in the context of previously published data, assessing the functional implications of an alternative architecture and effects for future studies.
\end{abstract}

Keywords: chemosensory array; chemotaxis; E. coli; cryo-electron tomography; molecular modelling

\section{Introduction}

Chemotactic responses in bacteria are mediated by large protein complexes known as chemosensory arrays, comprising thousands of copies of three primary components: transmembrane chemoreceptors (known as Methyl-accepting Chemotaxis Proteins or $\mathrm{MCPs})$, the CheA histidine kinase, and the CheW coupling protein [1]. Environmental cues received by the periplasmic domains of receptors initiate sensory signals that regulate CheA autophosphorylation activity, thereby modulating a cascade of intracellular phosphorylation reactions that culminate in adaptable control of the locomotor machinery [2]. The highly organised clustering of chemosensory proteins integrates complex chemical signals and dramatically enhances response cooperativity, facilitating the exquisite sensitivity and behavioural adaptation characteristic of chemotactic responses [3]. As such, the supramolecular array structure has been the subject of intense study, both as a model system for signal transduction and due to the involvement of chemotaxis in crucial biological processes such as cell adhesion [4], biofilm formation [4-6], bacterial symbiosis with plants [7] and pathogen infection of plant and human hosts [6,8-10].

First visualized by negative stain electron microscopy [11], the striking extended architecture of chemosensory arrays was immediately identified as an ideal target for cryoelectron microscopy [12] and cryo-electron tomography (cryo-ET) [13,14]. Early cryo-ET analyses revealed that chemoreceptors in a wide range of microbial species organise as receptor trimers of dimers (ToDs) that further pack into an extended hexagonal arrangement, which is considered to be their universal feature [15-17]. Subsequent cryo-ET studies, informed by crystal structures and molecular modelling, revealed the organisation of the baseplate region containing CheA and CheW in E. coli [18,19], describing the existence of six-membered (A.P5/W) 3 rings involving the CheA P5 regulatory domain (A.P5) and 
CheW that interlocked the cytoplasmic tips of receptor ToDs (Figure 1). Within this organisation, pairs of ToDs are linked by a CheA dimer and two CheW monomers to form core-signalling units (CSUs), the minimal complex required for receptor-mediated CheA regulation [20,21]. The CSU associates into a p6 symmetric lattice (i.e., displaying three-, and six-fold rotational symmetry in the centers of rings and two-fold rotational symmetry at the center of every CSU). In addition, $(\mathrm{W})_{6}$ rings composed exclusively of $\mathrm{CheW}$, which result from the addition of a flanking $\mathrm{CheW}$ to each ToD of a CSU, are proposed to further interconnect the p6 lattice $[19,22]$. Thus the general picture of chemosensory arrays that has emerged is that of an extended, pseudo-p6-symmetric lattice of interconnected CSU building blocks assembled on the inner membrane.
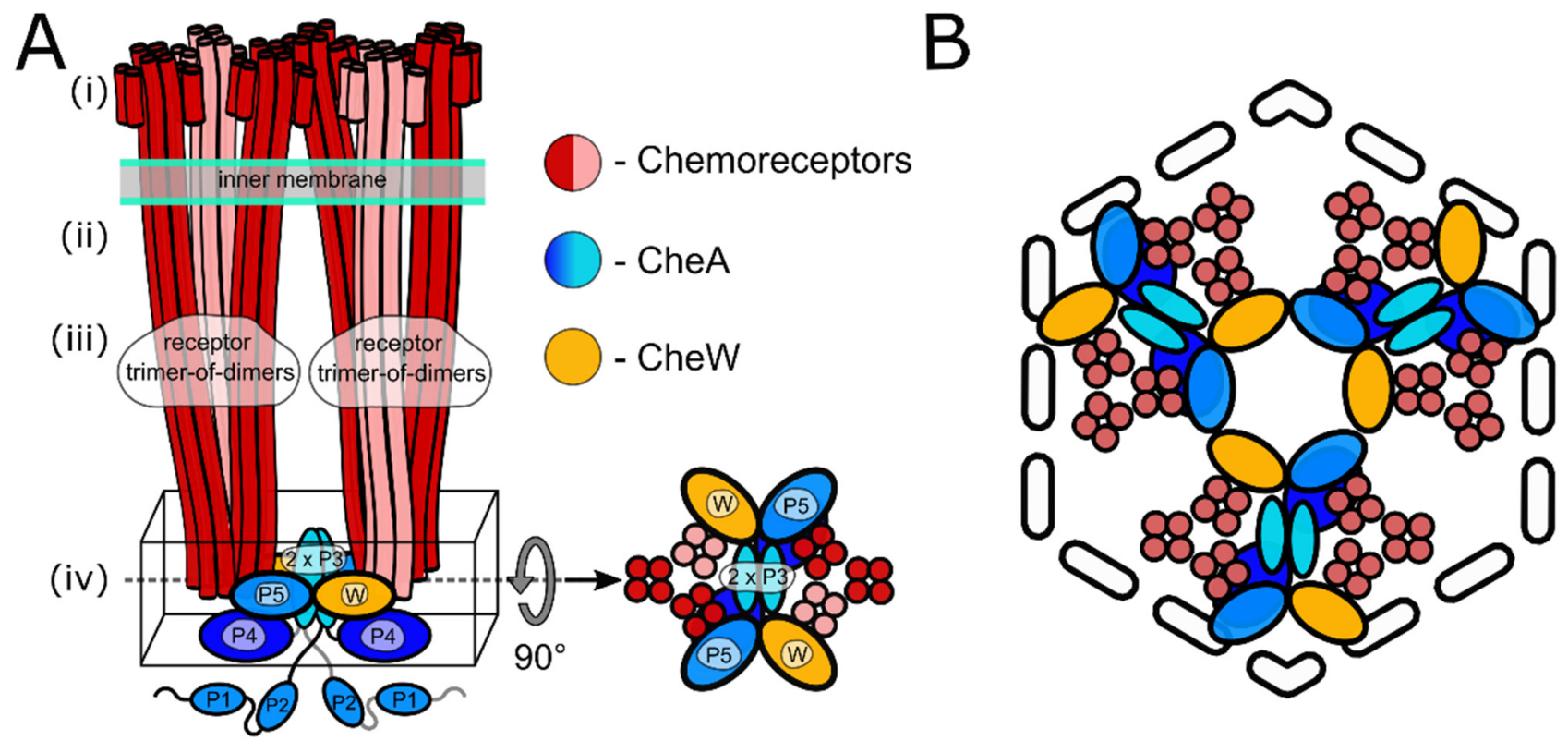

Core Signalling Unit

Figure 1. Schematics of the core signalling unit and organisation of three CSUs into a hexagon. (A) Two ToDs interact with CheA and CheW to form a CSU shown from the side. In each ToD, two MCP dimers are shown in red and one in salmon for perspective. CheA is shown in shades of blue, and CheW in gold. Domains of CheA are labelled. The baseplate region is boxed and also shown from the top. (B) Three CSUs assemble into a hexagon that gives rise to a (A.P5/W) 3 ring characteristic of the pseudo-p6-symmetric array architecture (see also Figure 3B for the extended array organisation showing the formation of $(\mathrm{W})_{6}$ rings).

Recent cryo-ET and molecular dynamics studies [23-25] have significantly increased the understanding of intra-CSU organisation and dynamics, culminating in the structure of a complete transmembrane CSU [23]. Although many questions regarding conformational rearrangements of the receptor and the kinase during signalling processes remain unanswered [2,26], even less is known about the ways in which signals are transmitted between CSUs. Generally speaking, analysis of array ultrastructure is complicated by limited long-range order in the structure, which is known to exhibit local deviations from an idealised symmetric architecture $[24,27,28]$ and can be assembled on membranes with varying degree of local curvature. Nevertheless, characterisation of the extended architecture of the chemosensory array is an essential step towards a molecular understanding of the cooperative allosteric interactions between array components that enable its unique capacity for efficient signal integration and amplification [2,3]. Here, we show that even the well-studied E. coli chemosensory array still holds surprises: the canonical pseudo-p6 organisation is not the only possible array architecture, nor does it adequately explain all existing experimental data. Instead, we highlight the existence of a pseudo-p2 organisation through cryo-ET observations of E. coli minicells. We propose molecular models of this 
alternate assembly as well as the canonical p6-symmetric organisation and compare their structural features in the light of current models of array structure and function.

\section{Methods}

\subsection{WM4196 Minicell-Producing Strain Culture}

WM4196 cells [23] were grown at $37^{\circ} \mathrm{C}$ in L broth supplemented with $34 \mu \mathrm{g} \mathrm{ml}{ }^{-1}$ chloramphenicol for $12 \mathrm{~h}$. Small volumes of this culture were used to inoculate larger volumes of $\mathrm{L}$ broth media (without antibiotics) to an initial OD600 value of 0.075 . These larger volume cultures were grown at $37^{\circ} \mathrm{C}$ for $4 \mathrm{~h}$ until final OD600 value of 1.75 . Details of the genetic characterisation, growth of the E. coli WM4196 strain, minicell separation from the mother cells, cryo-ET grid preparation and data acquisition are described in our previous manuscript [23].

\subsection{Tilt Series Alignment and Tomographic Reconstruction}

The acquired raw cryo-ET data, made available through the Electron Microscopy Public Image Archive (EMPIAR-10364), was reexamined in the present work. Multi-frame micrographs for each tilt image in EMPIAR-10364 were subject to whole frame alignment and image stacks were generated for each tilt-series in Warp. Tilt series were aligned automatically using the tilt-series alignment workflows available in Dynamo 1.1.478. Final bead positions from Dynamo were used to produce alignment parameters for the tilt-series with the IMOD program tiltalign, solving only for shifts and a constant tilt-axis rotation for the tilt-series with the robust fitting method. Tomograms were reconstructed based on these alignments in Warp.

\subsection{Denoising Tomograms}

Even and odd half-tomograms were generated with an isotropic voxel spacing of $5 \AA$ using Warp [29], from even and odd frames of multi-frame micrographs, respectively. A Noise2Noise [30] based denoising convolutional neural network was trained using cryo-CARE [31]. The cryo-CARE model was trained with a batch size of 16 , a learning rate of 0.0004 for 200 epochs with 75 training steps per epoch. The trained network was applied to the corresponding tomogram reconstructed from the full dataset to produce a denoised tomogram.

\subsection{Chemosensory Array Baseplate Segmentation and Visualisation}

Template matching of EMD-10160 in reconstructed tomograms with a voxel spacing of $17.96 \AA$ A was performed in the Dynamo software package [32], using both in-plane and out-of-plane sampling of 12 degrees. A set of cross-correlation peaks corresponding to the CSUs in the chemosensory array with a regular organisation were observed at a distance of $25 \mathrm{~nm}$ from intense cross-correlation response seen for the inner-membrane. A smooth, curved surface was modelled following this set of peaks as a membrane model in Dynamo. The mesh was exported, imposing consistent normal orientations, then imported with the corresponding tomogram (voxel spacing 17.96, reconstructed using the SIRT-like filter in IMOD with 15 iterations) into Membranorama for visualisation. Given that the EMPIAR10364 dataset contains six tilt series only, this procedure cannot be used for statistical evaluation on the relative prevalence of the $\mathrm{p} 2$ and $\mathrm{p} 6$ lattices but greatly facilitates visual examination of the array architecture.

\subsection{Molecular Modelling}

A model of the E. coli transmembrane CSU was constructed by extending a recent sub-nanometer resolution model of the baseplate region (PDB ID: 6S1K) [24] using the full-length E. coli Tsr ToD model derived in our previous manuscript [23]. Flanking CheW molecules were added to both bare receptors in the CSU model using the CheW/receptor binding mode observed in PDB ID 6S1K. Extended models for both the p2 and p6 symmetries were constructed by tiling their respective unit cells along the appropriate lattice 
vectors. In the case of the p2 lattice, the unit cell is the CSU itself, while in the p6 lattice, the unit cell consists of three CSUs arranged within a parallelepiped as previously described [22]. A lattice constant of $126 \AA$ was used in both cases as it produced an intact baseplate and is consistent with our previous measurements [23]. Modelling of the CheA.P1 and CheA.P2 domains was based on PDB ID 2LP4 [33], with missing residues in the P2-P3 linker filled in using Modeller v9.23 [34]. General modelling procedures and figure renderings were conducted using VMD v1.9.4 [35].

\section{Results and Discussion}

3.1. A Pseudo-p6 Symmetric Array Architecture Does Not Adequately Describe All Experimental Observations

Different strategies have been employed to obtain images of chemosensory arrays with the aim of improving both their interpretability and the results of subsequent subtomogram averaging experiments. These include (i) overexpression or derepression of array and /or flagellar genes to increase array size and occurrence frequency $[18,19,25]$, (ii) gentle cell lysis by a phage or an antibiotic to induce cytoplasmic leakage and thus reduce cell thickness [25,36-38], (iii) in vitro reconstitution on lipid monolayers from purified cytoplasmic components to obtain thin samples for high-resolution cryo-ET imaging [22,24], (iv) genetic manipulation of $E$. coli to express a single type of MCP, possibly with specific adaptation states or other mutations, thereby increasing array homogeneity and mimicking discrete signalling states [24,25], (v) exploration of the great variety of bacterial species [17], with often more complex and diverse chemotaxis systems, some of which are thinner than E. coli and (vi) use of bacterial minicells that bud near the cell poles where arrays are located $[19,23]$. Here, we re-examine the ultastructural context of our cryo-ET volumes of the E. coli WM4196 minicells which led to the complete in situ CSU structure [23] (EMPIAR-101364).

Side views of the E. coli chemosensory arrays have a characteristic comb-like appearance with MCP "teeth" protruding from the CheA/CheW baseplate located $30 \mathrm{~nm}$ under the inner membrane. The lines of MCPs extend all the way into the periplasm where, in the best cases, small globular densities corresponding to periplasmic domains are visible. Whereas such brush-like shapes can be directly seen in slices perpendicular to the direction of the electron beam in the tomographic reconstruction, and often even in projection images of the minicells, the higher-order organisation is easier to infer from top views, in which the array baseplate and its hallmark honeycomb pattern is oriented perpendicular to the optical axis. We leveraged the cryo-CARE (Context-Aware image Restoration) method for Noise2Noise-based tomogram denoising [31], a technique which both improves contrast and reduces the appearance of missing wedge artefacts in tomographic reconstructions, to better visualize the chemoreceptor arrays in our low signal-to-noise tomograms of the E. coli WM4196 minicells (EMPIAR-101364) [23]. Unexpectedly, whilst examining arrays in denoised tomograms in which receptors were aligned both perpendicular (Figure 2A) and parallel (Figure 2B) to the electron beam during imaging, we did not observe regions with an unequivocal p6-symmetry, displaying obvious 3- or 6-fold symmetry axes. Instead, they contained a repeating diamond-shaped motif arranged in a p2-symmetric fashion. Given CSU stability, biochemical necessity and the CSU reconstruction derived from these data, we postulate that the observed diamond shaped motif corresponds to a CSU (Figure 2C). 


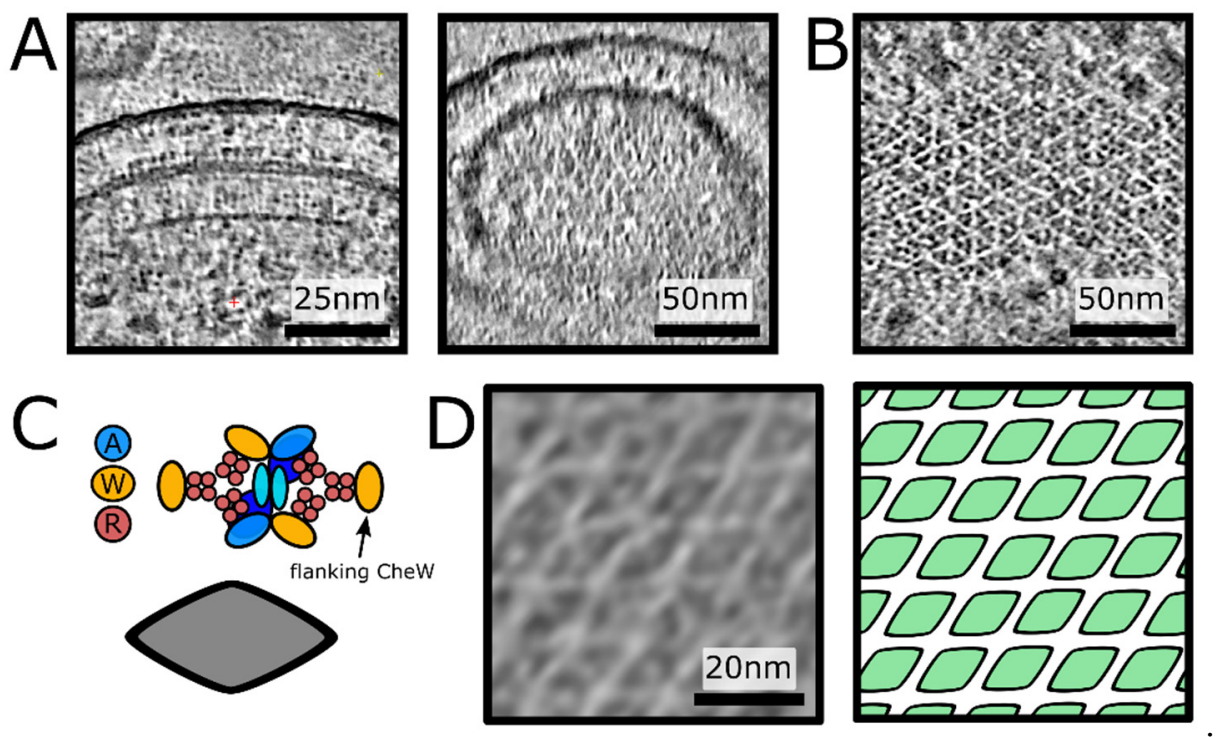

Figure 2. Direct visualisation of a p2 organisation of core-signalling units in an E. coli minicell strain. (A) 10nm thick oblique slices through a denoised tomogram with a chemoreceptor array aligned with the optical axis of the microscope. Scale bar $25 \mathrm{~nm}$. (B) 10nm thick oblique slice through a denoised tomogram with a chemoreceptor array aligned perpendicular to the optical axis of the microscope. Scale bar $50 \mathrm{~nm}$. (C) A schematic of the CSU (top) showing the positions of CheA (blue), CheW (yellow) and receptor proteins (red). A simplified visualisation of the CSU is shown as a grey diamond (bottom). (D) The chemoreceptor array from (B), depicted as a membranogram (left) following the curved surface of the array inside the cell, shows a p2 symmetric array of CSUs (right). The protein density in A, B and D is black. Scale bar $20 \mathrm{~nm}$.

Surprised by this observation, we decided to visualize the organisation with Membranorama, a tool which allows projection of tomographic density onto an arbitrary curved 3D surface instead of simple oblique slices [39]. Making use of the Dynamo software package [32], we performed template matching in the minicell tomograms using our reference array structure (EMD-10160). The resulting cross-correlation volume enabled accurate definition of a 3D surface following the intrinsic curvature of the array, onto which we projected local tomographic density from a SIRT-like filtered tomogram (see Methods). Dynamic exploration of the 3D surface, shifting the region of density projected along the surface normal, shows a pseudo-p2-symmetric assembly of CSUs in situ (Figure 2D, Figure S1). The resulting surface projections are best inspected directly in 3D (Supplementary Movie S1), enabling simultaneous examination of the entire in situ array organisation in one of our E. coli WM4196 minicell tomograms where the pseudo-p2-symmetry is particularly evident.

Strictly speaking one should refer to a "pseudo-symmetry" when describing a 3D organisation on a curved membrane surface and use the term symmetry only for $2 \mathrm{D}$ lattices. However, in the remainder of this paper we will refer to the array architecture as either p2-symmetric or p6-symmetric for the sake of simplicity. It is critical to note here that the WM4196 minicells analysed in this study possess arrays with normal stoichiometries of chemosensory components, and include a native distribution of MCPs that presumably have heterogeneous adaptation states. Thus, observed structural differences cannot be attributed to the genetic manipulation of the array components.

\subsection{Molecular Models of p2- and p6-Symmetric Array Architectures}

To account for and characterise the differences between the p2- and p6-symmetric array architectures at the individual-protein level we constructed a molecular model of each lattice (Figures 3 and 4, Supplementary Movie S2). 

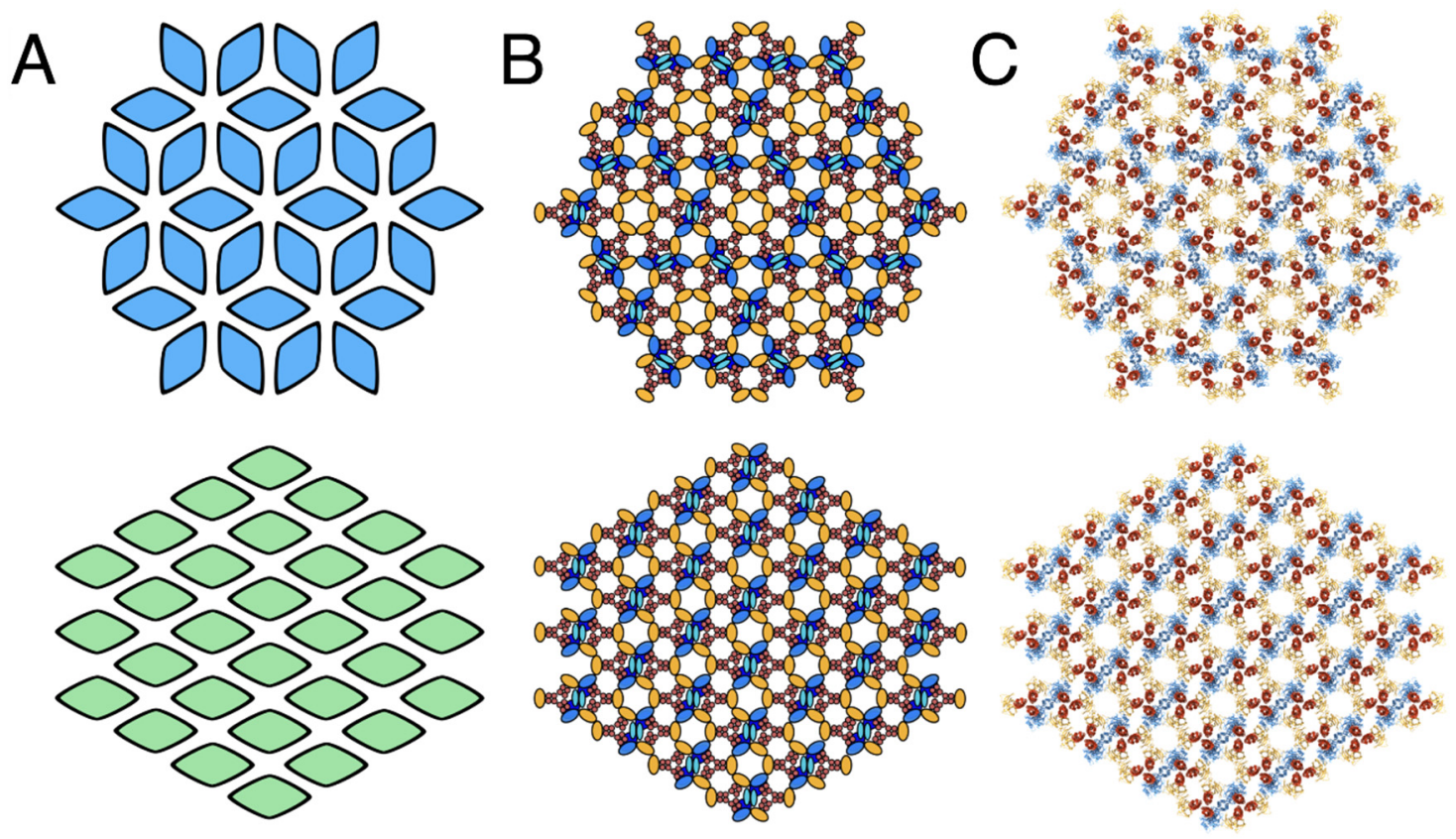

Figure 3. Schematics and models of p6 and p2 chemosensory array architectures. (A) Simplified schematics of p6 (top, blue) and p2 (bottom, green) array architectures with each diamond representing a CSU. (B) Schematics of p6 (top) and p2 (bottom) array architectures in which CheA, CheW and receptor proteins are depicted and coloured blue, green and red, respectively. (C) The baseplate region of all-atom models of the p6 (top) and p2 (bottom) array architectures.
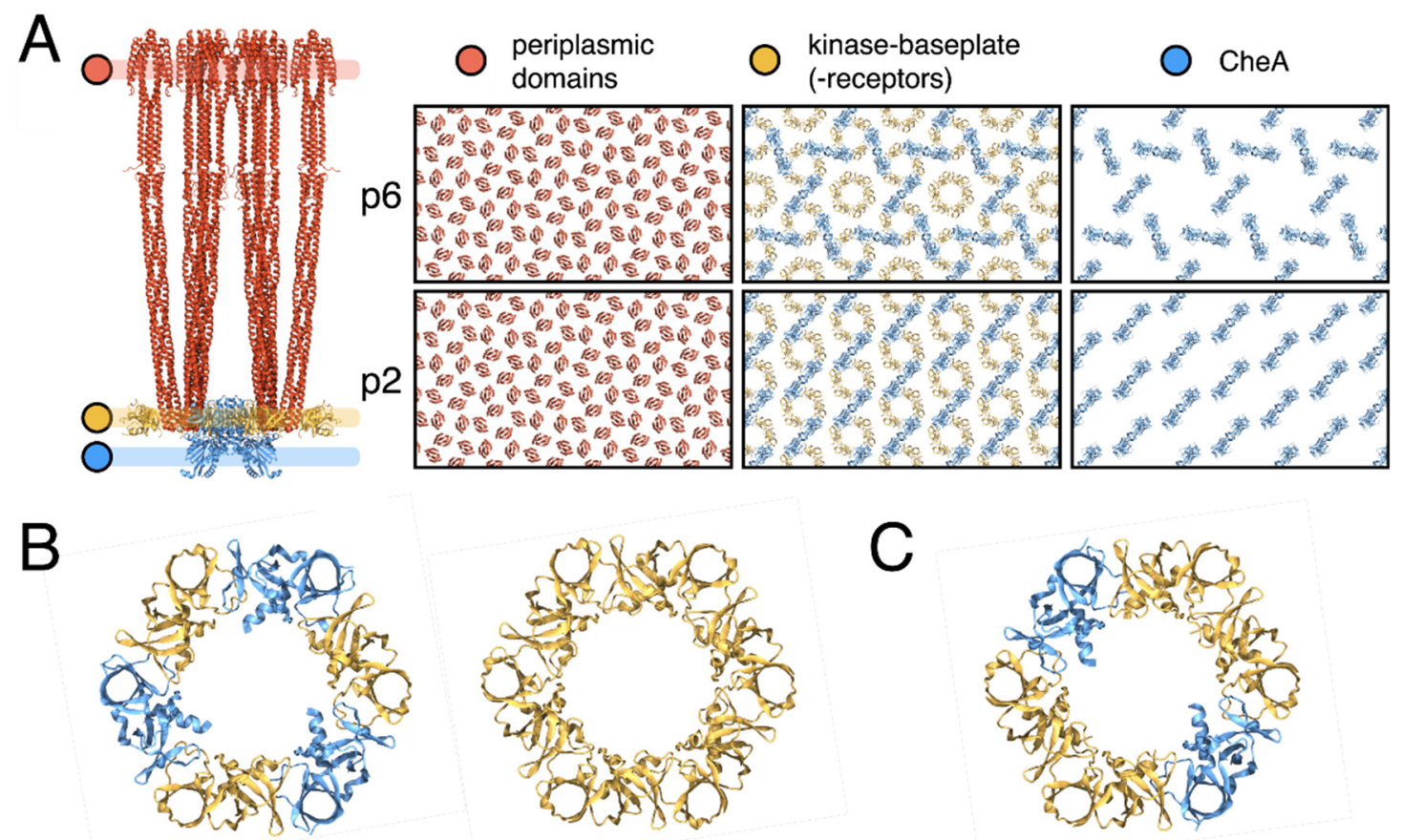

Figure 4. Structural features of p6 and p2 chemosensory array architectures. (A) All-atom model of the CSU (left) including flanking $\mathrm{CheW}$ molecules. Regions corresponding to the periplasmic domains of receptor proteins, kinase baseplate and CheA.P4 are demarcated in orange, yellow and blue, respectively. The corresponding regions in both the p6 and p2 array architectures are presented to the right, showing the near-identical receptor organisation and structural differences in the baseplate region. (B) The $(\mathrm{W})_{6}$ and $(\mathrm{A} . \mathrm{P} 5 / \mathrm{W})_{3}$ rings of CheA and CheW present in the p6 array architecture. (C) The (A.P5/W/W $)_{2}$ ring of CheA and $\mathrm{CheW}$ present in the 2 array architecture. 
To this end, we first made a model for the full-length E. coli CSU by extending a recent structure of the CSU baseplate (PDB ID: 6S1K) [24] and using the full-length E. coli Tsr receptor ToD coordinates derived in our previous manuscript [23] Models for both architectures were then constructed by tiling the CSU in accordance with the appropriate lattice vectors and using a lattice constant of $126 \AA$ [23]. As expected, both models reproduced the universal hexagonal arrangement of receptor ToDs. In addition, at the level of the kinase baseplate, the p6 model contained both the anticipated (A.P5/W) 3 rings and empty sites for $(\mathrm{W})_{6}$ rings. In contrast, the $\mathrm{p} 2$ model possessed only a single type of semi-formed ring whereby two CSUs provide a (A.P5/W) pair and two opposing CSUs present a bare receptor dimer. Addition of flanking CheW monomers to each CSU filled the empty $(\mathrm{W})_{6}$ rings in the $\mathrm{p} 6$ model and gave rise to complete, two-fold symmetric (A.P5/W/W $)_{2}$ rings in the $\mathrm{p} 2$ model. Thus while the flanking CheW molecules are involved in coupling neighbouring CSUs through rings in both lattices, their exact role is symmetrydependent. In the p6 model, flanking CheWs serve to reinforce an existing lattice created by the $(\mathrm{A} . \mathrm{P} 5 / \mathrm{W})_{3}$ rings formed between three CSUs, whereas in the $\mathrm{p} 2$ model they are essential to the formation of an extended $\mathrm{p} 2$ lattice, which requires the interaction of four CSUs with flanking CheWs to produce an intact baseplate. Another striking difference between the two architectures concerns the intermolecular organisation of CheA (Figure 4, Movie S2). In the p6 organisation, CheA molecules are arranged in a trimeric fashion with one monomer of each CheA dimer contributing a P5 domain to a (A.P5/W) 3 ring in the center of the trimer, and the other monomer contributing a P5 domain to one of the three surrounding (A.P5/W) 3 rings. These trimeric CheA arrangements are themselves organised in an interlocking hexameric fashion around central $(\mathrm{W})_{6}$ rings. In the p2 organisation, however, CheA dimers form parallel stripes such that each monomer of the CheA dimer contributes its $\mathrm{P} 5$ domains to an opposite (A.P5/W/W $)_{2}$ ring, resulting in chains of interlocked rings. Interestingly, this difference in CheA arrangement alters considerably both the intermolecular distance and the relative orientation of neighbouring CheA molecules, the potential consequences of which are discussed further below.

The proposed $\mathrm{p} 2$ architecture does not require any deviation from the current understanding of CSU structure and preserves all critical intra-CSU signalling interfaces between receptor dimers, CheA.P5 and CheW. In addition, despite the aforementioned differences in overall baseplate organisation, the same three types of interfaces are exclusively present within the baseplate rings of both lattices. These include the previously characterized interface I, involving subdomain 1 of CheA.P5 and subdomain 2 of CheW [40], and interface II, involving the subdomain 2 of CheA.P5 and subdomain 1 of CheW [41], as well as an interface involving subdomain 1 and subdomain 2 of two CheW monomers, which we term interface III (Figures $4 \mathrm{~B}$ and 5). Assuming fully interconnected p2 and p6 lattices (i.e., with all flanking $\mathrm{CheW}$ sites occupied), the relative abundances of ring interfaces are also identical within each lattice, namely $2 \times$ interface I, $4 \times$ interface II, and $4 \times$ interface III (Figure 5). The differences in ring structure are therefore primarily manifest as a spatial redistribution of the baseplate ring interfaces. Whilst in the p6 lattice, interfaces I and II alternate within the (A.P5/W $)_{3}$ rings and interface III is present exclusively within the $(\mathrm{W})_{6}$ rings, in the $\mathrm{p} 2$ lattice, all three interface types are present within each (A.P5/W/W) ring and each type is adjacent to the other two (Figure 5). Notably, the extended p2 and p6 molecular models yield no indication that interfaces I, II, or III should be subject to different structural constraints within the two lattices. For example, interface II is expected to possess interactions between roughly the same subset of interfacial residues in both the p6 and p2 lattices despite utilizing a core $\mathrm{CheW}$ in the former and a flanking $\mathrm{CheW}$ in the latter. 
A
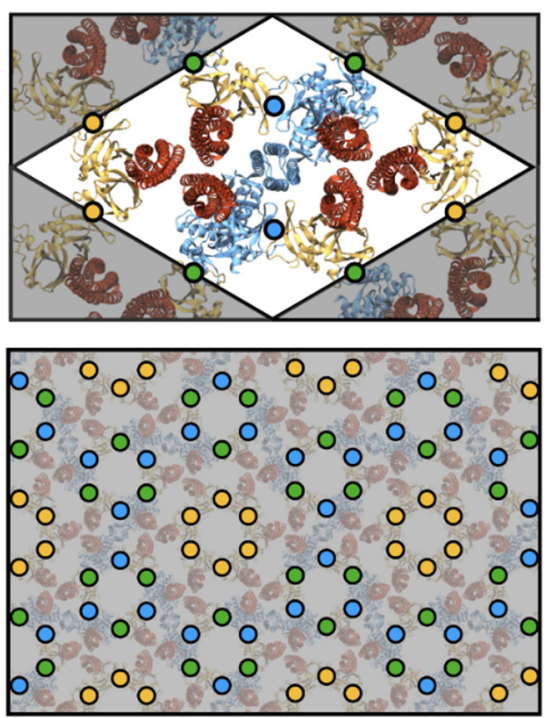
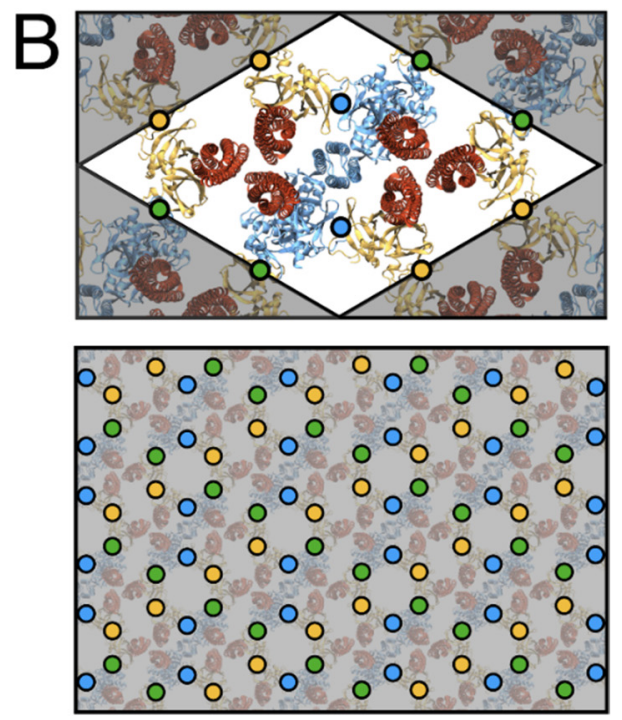

Interface I (A.P5/W intra-CSU)

O Interface II (A.P5/W inter-CSU)

Interface III (W/W inter-CSU)

Figure 5. Assembly interfaces of p6 and p2 chemosensory array architectures. (A) The p6 array architecture. (B) The p2 array architecture. The positions of assembly interfaces I (A.P5/W intra-CSU), II (A.P5/W inter-CSU) and III (W/W inter-CSU) are depicted in blue, green and yellow, respectively. For each architecture the spatial distribution is depicted around one CSU (top) and a larger assembly of CSUs (bottom).

\subsection{Structure Based-Analysis of Functional Implications of $p 2$ Architecture}

Given that the foremost distinguishing feature between the $\mathrm{p} 2$ architecture reported here and the canonical p6 architecture is their respective inter-CSU organisations, one might expect that signalling properties arising due to the interactions between CSUs would be affected. Following the elucidation of the p6 architecture in E. coli $[18,19]$, structural lesions designed to affect the allosteric coupling between CSUs through disruption of interface II were shown to have dramatic effects on the cooperativity and sensitivity of the chemotactic response, suggesting that these properties were directly linked to the degree of interconnectedness between CSUs [41,42]. This notion has been further advanced by a recent study investigating the detailed role of the $(\mathrm{W})_{6}$ rings within the $\mathrm{p} 6$ architecture, showing that the cooperativity of the signalling response increases with the number and completeness of $(\mathrm{W})_{6}$ rings, which vary widely depending on array assembly conditions (Piñas et al., personal communication). Given that the baseplate connectivity within the p6 lattice depends on the degree of $(\mathrm{W})_{6}$ ring occupancy, one might therefore expect the p2 architecture, which necessarily exhibits a fully interconnected baseplate, to possess a higher degree of inherent cooperativity. An analysis of the number of interfaces required to get from each receptor within a given CSU to the nearest CheA in both organisations additionally suggests that signals might be more readily transmitted between neighbouring components via baseplate rings in the $\mathrm{p} 2$ architecture. Indeed, all three receptors in a given ToD are within two interfaces from the nearest CheA.P5 in the $\mathrm{p} 2$ organisation, whereas only two receptors are within this distance in the p6 architecture and the third receptor is bound to a ring that does not contain CheA at all (Figure 5). However, such analysis is complicated, especially over long distances, by the fact that specific baseplate interfaces and/or ring types could differ in flexibility and dynamics owing to their composition, which might change the efficacy of signal transmission between CSUs. An interesting corollary to the observed baseplate organisation is that signalling within the CSU itself may also be altered despite its conserved structure. Specifically, there is evidence of 
functional asymmetries between receptors within a ToD depending on the particular baseplate component to which they are attached $[25,43]$. Thus the noted alterations in the structural context of each baseplate interface might cause such receptor symmetry breaking to manifest differently within the two lattices despite the conserved hexagonal arrangement of receptors (Figure S2).

Finally, differences in both the intermolecular distances and relative orientations of neighbouring CheA dimers may also have non-trivial effects on signalling and cooperativity. The P1 and P2 domains of CheA, which mediate the transfer of phosphoryl groups between CheA.P4 and the response regulator CheY, reside below the baseplate layer and are connected to each other and CheA.P3 by long, unstructured linkers. While, as far as we are aware, the possibility of inter-dimer CheA communication within chemosensory arrays has neither been proposed nor ruled out elsewhere, our models suggest that these linkers could allow interactions between the P1 domain of a given CheA and a P4 domain of multiple neighbouring CheAs (Figure 6). Such long-range CheA interactions may, therefore, contribute to cooperative array signalling and would likely be altered by the global change in CheA organisation between the p6 and p2 lattices. Ultimately, the answer to these questions will require a thorough investigation of cooperativity within arrays with different, well-defined lattice connectivity. Such an investigation should be possible through an application of the present tools to image appropriately engineered arrays in combination with emerging single-cell FRET experiments to quantify signalling responses $[41,42,44]$.

A

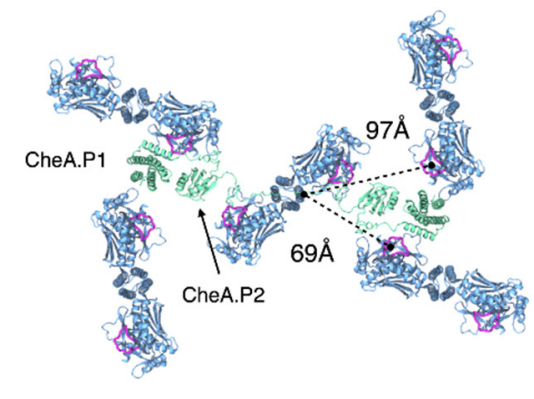

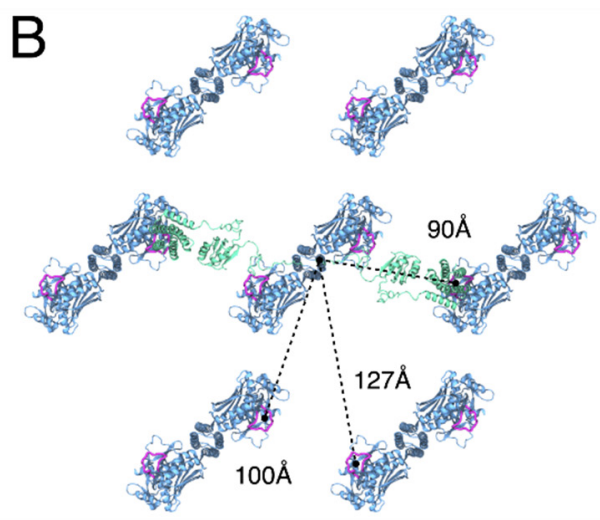

Figure 6. Difference in the intermolecular distance and the relative orientation of neighbouring CheA molecules between $\mathrm{p} 6$ and $\mathrm{p} 2$ chemosensory array architectures. (A) The p6 architecture. (B) The p2 architecture. Rough distances between a given CheA monomer, as measured from the end of the P2-P3 linker (residue I264), and ATP-binding sites on neighbouring CheAs that may be accessible in a trans-fashion by the associated P1 domain (dashed lines). The CheA.P1 and CheA.P2 domains are modelled for a centralized CheA dimer and shown in green. The ATP lid (residues 455-475) of each CheA monomer is shown in purple. Receptors, CheW, and CheA.P5 are not shown for clarity.

\subsection{Implications of the Observation of p2-Symmetric Chemosensory Arrays in E. coli}

The bulk of cryo-ET imaging of chemosensory arrays in diverse biological contexts demonstrates a clear preference for the formation of a p6 symmetric architecture in E. coli. The question thus emerges: what are the molecular origins of the $\mathrm{p} 2$ symmetric architecture seen in this study? Interestingly, a recent publication by Muok et al. describes a p2 chemosensory array organisation in the spirochete Treponema denticola [45], which exhibits a linear CheA arrangement, including rings involving interactions between a classical $\mathrm{CheW}$ and a spirochete-specific CheW variant that are analogous to the (A.P5/W/W $)_{2}$ rings seen in our model. Due to the orientation of the linear CheA strands, which always appear to run parallel to the cell axis, and because of the very high curvature of these cells perpendicular to the cell axis, the authors propose that the array organisation seen 
in T. denticola evolved specifically to accommodate the spirochetes' high curvature. This notion is further supported by the presence of unique structural features in the CheW variant and CheA dimerisation domain, which are suggested to be critical for maintaining the structural integrity and function of these highly curved arrays. Thus, considering the T. denticola array organisation, it is tempting to ascribe our observation of a p2 organisation in E. coli minicells to their increased curvature relative to standard E. coli cells. However, it should be noted that the E. coli minicells studied here are considerably less curved than the T. denticola cells. Assuming an initially spherical minicell geometry (i.e., before plunge freezing), we estimate the average radius of curvature of the inner membrane to be $153 \mathrm{~nm}$ (Figure S3) as compared to $28 \mathrm{~nm}$ reported for the T. denticola cells. Moreover, given the well-documented stability of the chemosensory array both in vivo and in vitro [46-49], it is likely that the p2 organisation is present as such in certain WM4196 mother cells, which are necessarily less curved, prior to minicell budding. Intriguingly, re-examination of previously published data [24] in light of these new observations appears to indicate the presence of $\mathrm{p} 2$ architectures even in arrays of purified E. coli cytoplasmic components reconstituted on lipid monolayers with very low curvature (Figure S4).

One of the means of regulation of the array assembly into a p2 or p6-symmetric architecture may also originate from the assembly dynamics. The importance of the relative expression levels of array components for the formation of extended, well-ordered array patches in vitro and in situ is well documented $[17,22,27]$. It is possible therefore that the p2 architecture may arise via an alternative assembly pathway, involving alterations in spatio-temporal regulation of component expression. Although a detailed array assembly mechanism remains elusive, the current working model in E. coli suggests that receptors first form ToDs that aggregate near the cellular poles, where they combine with CheA and $\mathrm{CheW}$ to form complete CSUs, which associate further into intermediate extended structures. While the canonical p6 architecture may accommodate either filled, partially filled or empty $(\mathrm{W})_{6}$ rings at the six-fold symmetry axes of this arrangement, the p2 organisation presented here exhibits only identical nodes of (A.P5/W/W $)_{2}$ rings. Considering that the flanking CheW molecules, which are not necessary for formation of a p6 lattice, take on a critical role in the $\mathrm{p} 2$ architecture, we propose an assembly pathway in which increased occupancy of these flanking positions on CSUs increases the probability of forming p2-symmetric patches. Presumably, the p2 pathway becomes more important upon increase of the local concentration of CheW during the early stages of array formation. The preponderance of the p6 architecture may therefore simply result from a more favourable assembly pathway. Interestingly, in many bacteria the $\mathrm{CheW}$ :CheA ratio is much higher than in E. coli [17]. An intriguing possibility is that the $\mathrm{p} 6$ and $\mathrm{p} 2$-symmetric architectures may compete during the formation of the extended array, similar to what has been observed in some bacterial S-layers [50]. Within such a phase-competition picture, it may be that intermediate-curvature settings tip the balance in favour of a 2 organisation, which becomes a structural necessity in the face of extreme curvatures, such as in T. denticola as suggested by Muok et al. Additional work will be required to identify how specific environmental factors contribute to array assembly and to unravel how their interplay affects array function.

As a final note, the observation of p2 patches elsewhere would imply that it may be present more broadly within previously analysed datasets, but has gone unnoticed. A possible reason for this is the use of symmetrisation during subtomogram averaging experiments. Indeed, large portions of the overall structure remain similar upon 2-, 3and 6-fold symmetrisation (Figure S5), a property which has historically been used to improve reconstructions from small numbers of subtomograms. In an effort to enable further analysis of baseplate asymmetries, we have deposited our raw cryo-ET data for WM4196 minicells in the Electron Microscopy Data Bank (EMPIAR-10364) and would like to urge others to make available their raw data for previously published work.

In summary, we show that the $\mathrm{p} 6$ architecture does not adequately explain all images of $E$. coli minicells with classical chemotaxis proteins and propose an alternative which 
respects the observed p2 symmetry as well as the current understanding of CSU structure, including previously characterised signalling interfaces. Whilst the physiological reasons for the existence of two distinct types of array architecture with possibly differing signalling properties are as yet unknown, the discovery of alternative assemblies and their probable coexistence within collected datasets should undoubtedly stimulate further investigation and influence the way biochemical and structural data from chemotactic systems are analysed moving forward.

\section{Reporting Summary}

Further information on research design is available in the Nature Research Reporting Summary linked to this article.

Supplementary Materials: The following are available online at https:/ / www.mdpi.com/2218-2 73X/11/4/495/s1, Figure S1: Images of chemosensory arrays in WM4196 minicells (A) Membranograms of array patches in our dataset generated from SIRT-like filtered tomograms. Scale bars are not provided because the images are showing data projected on curved surfaces. (B) Oblique $10 \mathrm{~nm}$ thick slices through denoised tomograms containing arrays with MCPs aligned parallel to (top left) and perpendicular to (bottom left) the optical axes. Their corresponding power spectra are shown on the right. Figure S2: The hexagonal arrangement of receptors is conserved between the p6 and p2 array architectures. Top view of the p6- and p2-symmetric array models with receptor periplasmic domains coloured according to baseplate partner. Receptors bound to CheA.P5 are shown in red, core CheW in yellow, and flanking CheW in blue. Figure S3: Estimation of the average radius of curvature of the inner membrane of a WM4196 minicell. An XZ projection through the region of a tomogram containing a p2-symmetric array architecture. Local estimates for radii of curvature are indicated on an overlaid ellipse. A circle with curvature equal to the mean curvature of the ellipse is plotted as a dashed line. Figure S4: An image region of an in vitro reconstituted chemosensory array with very low curvature taken from [24]. Image regions which appear to display a p2 array architecture are highlighted in dashed rectangles. Scale $50 \mathrm{~nm}$. Figure S5: The effects of applying different symmetries to reconstructions centered at the center of six receptor trimers-of-dimers in both p6 and p2 architectures. Applying symmetry during subtomogram averaging experiments on particles centered at the centeres of receptor trimer-of-dimers will perturb the structure differently depending on which ring structure is present in the data. In the $\mathrm{p} 6$ array architecture the $(\mathrm{W} / \mathrm{A})_{3}$ ring is 3-fold symmetric, respecting only $\mathrm{C} 1$ and $\mathrm{C} 3$ symmetries while the $(\mathrm{W})_{6}$ ring is 6-fold symmetric, respecting $\mathrm{C} 1, \mathrm{C} 2, \mathrm{C} 3$ and $\mathrm{C} 6$ symmetries. The $(\mathrm{W} / \mathrm{W} / \mathrm{A})_{2}$ ring in the $\mathrm{p} 2$ array architecture is 2-fold symmetric, respecting only $\mathrm{C} 1$ and $\mathrm{C} 2$ symmetries. The only symmetry common to all ring structures from both p2 and p6 architectures is C1. Movie S1: Dynamic exploration of a tomogram on a curved surface in the region of the chemosensory array. Density from a WM4196 tomogram is projected onto a curved surface in the tomogram at the level of the chemosensory array inside the minicell. The region of density projected is shifted along the normal to the surface of each triangle in the mesh to explore the tomogram at different levels of the array architecture. Movie S2: A comparison of models of p6 and p2 chemosensory array architectures. The models of both p6 and p2 symmetric array architectures are visualised as slices at different levels of the structure to highlight similarities and differences at various positions in the extended architecture. The arrow on the model of the chemosensory array on the left hand side indicates the position of the slice through the model of the extended architectures displayed on the right hand side.

Author Contributions: A.B. analysed cryo-ET data with input from I.G., C.K.C. and A.B. constructed the model of the 2 architecture in discussion with I.G. and P.J.S., A.B. and C.K.C. prepared the figures and movies. I.G. supervised the study. I.G., A.B. and C.K.C. wrote the manuscript. Correspondence and request for materials should be addressed to I.G. All authors have read and agreed to the published version of the manuscript.

Funding: This research was funded by European Union's Horizon 2020 research and innovation programme, grant number 647784, and by the U.K. Biotechnology and Biological Sciences Research Council, grant number BB/S003339/1.

Data Availability Statement: The raw data from which tomograms were calculated, as well as reconstructed tomograms, are available on EMPIAR with accession code EMPIAR-10364. The Cryo- 
ET map derived from these tilt series and published in [23] is available from the EMDB with accession code EMD-10160. Coordinates for both the p2- and p6-symmetric E. coli array models are available for download at doi:10.5281/zenodo.4302473.

Acknowledgments: We acknowledge Diamond Light Source for access and support of the cryo-EM facilities at the UK's national Electron Bio-imaging Centre (eBIC), funded by the Wellcome Trust, MRC and BBRSC. Cryo-ET data acquisition has been supported by iNEXT, grant number 653706 (PID:2626), funded by the EU Horizon 2020 programme. We are particularly grateful to Daniel Castaño-Diez for help and discussions on image processing and for development of Dynamo. AB is supported by a University Grenoble Alpes PhD fellowship and by a Fondation pour la Recherche Medicale (FRM) fellowship FDT202001011069.

Conflicts of Interest: The authors declare no competing interests.

\section{References}

1. Falke, J.J.; Piasta, K.N. Architecture and signal transduction mechanism of the bacterial chemosensory array: Progress, controversies, and challenges. Curr. Opin. Struct. Biol. 2014, 29, 85-94. [CrossRef]

2. Parkinson, J.S.; Hazelbauer, G.L.; Falke, J.J. Signaling and sensory adaptation in Escherichia coli chemoreceptors: 2015 update. Trends Microbiol. 2015, 23, 257-266. [CrossRef]

3. Hazelbauer, G.L.; Falke, J.J.; Parkinson, J.S. Bacterial chemoreceptors: High-performance signaling in networked arrays. Trends Biochem. Sci. 2008, 33, 9-19. [CrossRef] [PubMed]

4. Laganenka, L.; Colin, R.; Sourjik, V. Chemotaxis towards autoinducer 2 mediates autoaggregation in Escherichia coli. Nat. Commun. 2016, 7, 12984. [CrossRef] [PubMed]

5. Alexandre, G. Chemotaxis control of transient cell aggregation. J. Bacteriol. 2015, 197, 3230-3237. [CrossRef] [PubMed]

6. He, K.; Bauer, C.E. Chemosensory signaling systems that control bacterial survival. Trends Microbiol. 2014, 22, 389-398. [CrossRef]

7. Scharf, B.E.; Hynes, M.F.; Alexandre, G.M. Chemotaxis signaling systems in model beneficial plant-bacteria associations. Plant Mol. Biol. 2016, 90, 549-559. [CrossRef]

8. Gotoh, Y.; Eguchi, Y.; Watanabe, T.; Okamoto, S.; Doi, A.; Utsumi, R. Two-component signal transduction as potential drug targets in pathogenic bacteria. Curr. Opin. Microbiol. 2010, 13, 232-239. [CrossRef]

9. Johnson, K.S.; Ottemann, K.M. Colonization, localization, and inflammation: The roles of H. pylori chemotaxis in vivo. Curr. Opin. Microbiol. 2018, 41, 51-57. [CrossRef]

10. Matilla, M.A.; Krell, T. The effect of bacterial chemotaxis on host infection and pathogenicity. FEMS Microbiol. Rev. 2018, 42. [CrossRef]

11. Weis, R.M.; Hirai, T.; Chalah, A.; Kessel, M.; Peters, P.J.; Subramaniam, S. Electron microscopic analysis of membrane assemblies formed by the bacterial chemotaxis receptor Tsr. J. Bacteriol. 2003, 185, 3636-3643. [CrossRef] [PubMed]

12. Zhang, P.; Bos, E.; Heymann, J.; Gnaegi, H.; Kessel, M.; Peters, P.J.; Subramaniam, S. Direct visualization of receptor arrays in frozen-hydrated sections and plunge-frozen specimens of E. coli engineered to overproduce the chemotaxis receptor Tsr. J. Microsc. 2004, 216, 76-83. [CrossRef] [PubMed]

13. Briegel, A. Strukturuntersuchungen an Prokaryonten mit Kryoelektronentomographie. Ph.D. Thesis, Technische Universität München, Munich, Germany, 2005.

14. Zhang, P.; Khursigara, C.M.; Hartnell, L.M.; Subramaniam, S. Direct visualization of Escherichia coli chemotaxis receptor arrays using cryo-electron microscopy. Proc. Natl. Acad. Sci. USA 2007, 104, 3777-3781. [CrossRef] [PubMed]

15. Briegel, A.; Ortega, D.R.; Tocheva, E.I.; Wuichet, K.; Li, Z.; Chen, S.; Müller, A.; Iancu, C.V.; Murphy, G.E.; Dobro, M.J.; et al. Universal architecture of bacterial chemoreceptor arrays. Proc. Natl. Acad. Sci. USA 2009, 106, 17181-17186. [CrossRef]

16. Briegel, A.; Ortega, D.R.; Huang, A.N.; Oikonomou, C.M.; Gunsalus, R.P.; Jensen, G.J. Structural conservation of chemotaxis machinery across Archaea and Bacteria. Environ. Microbiol. Rep. 2015, 7, 414-419. [CrossRef] [PubMed]

17. Yang, W.; Briegel, A. Diversity of bacterial chemosensory arrays. Trends Microbiol. 2020, 28, 68-80. [CrossRef]

18. Briegel, A.; Li, X.; Bilwes, A.M.; Hughes, K.T.; Jensen, G.J.; Crane, B.R. Bacterial chemoreceptor arrays are hexagonally packed trimers of receptor dimers networked by rings of kinase and coupling proteins. Proc. Natl. Acad. Sci. USA 2012, 109, 3766-3771. [CrossRef] [PubMed]

19. Liu, J.; Hu, B.; Morado, D.R.; Jani, S.; Manson, M.D.; Margolin, W. Molecular architecture of chemoreceptor arrays revealed by cryoelectron tomography of Escherichia coli minicells. Proc. Natl. Acad. Sci. USA 2012, 109, E1481-E1488. [CrossRef] [PubMed]

20. Li, M.; Hazelbauer, G.L. Core unit of chemotaxis signaling complexes. Proc. Natl. Acad. Sci. USA 2011, 108, 9390-9395. [CrossRef]

21. Li, M.; Khursigara, C.M.; Subramaniam, S.; Hazelbauer, G.L. Chemotaxis kinase CheA is activated by three neighbouring chemoreceptor dimers as effectively as by receptor clusters. Mol. Microbiol. 2010, 79, 677-685. [CrossRef]

22. Cassidy, C.K.; Himes, B.A.; Alvarez, F.J.; Ma, J.; Zhao, G.; Perilla, J.R.; Schulten, K.; Zhang, P. CryoEM and computer simulations reveal a novel kinase conformational switch in bacterial chemotaxis signaling. eLife 2015, 4, 1-20. [CrossRef]

23. Burt, A.; Cassidy, C.K.; Ames, P.; Bacia-Verloop, M.; Baulard, M.; Huard, K.; Luthey-Schulten, Z.; Desfosses, A.; Stansfeld, P.J.; Margolin, W.; et al. Complete structure of the chemosensory array core signalling unit in an E. coli minicell strain. Nat. Commun. 2020, 11, 1-9. [CrossRef] 
24. Cassidy, C.K.; Himes, B.A.; Sun, D.; Ma, J.; Zhao, G.; Parkinson, J.S.; Stansfeld, P.J.; Luthey-Schulten, Z.; Zhang, P. Structure and dynamics of the E. coli chemotaxis core signaling complex by cryo-electron tomography and molecular simulations. Commun. Biol. 2020, 3, 1-10. [CrossRef]

25. Yang, W.; Cassidy, C.K.; Ames, P.; Diebolder, C.A.; Schulten, K.; Luthey-Schulten, Z.; Parkinson, J.S.; Briegel, A. In Situ conformational changes of the Escherichia coli serine chemoreceptor in different signaling states. mBio 2019, 10, e00973-19. [CrossRef]

26. Muok, A.R.; Briegel, A.; Crane, B.R. Regulation of the chemotaxis histidine kinase CheA: A structural perspective. Biochim. Biophys. Acta Biomembr. 2020, 1862, 183030. [CrossRef] [PubMed]

27. Briegel, A.; Ladinsky, M.S.; Oikonomou, C.; Jones, C.W.; Harris, M.J.; Fowler, D.J.; Chang, Y.W.; Thompson, L.K.; Armitage, J.P.; Jensen, G.J. Structure of bacterial cytoplasmic chemoreceptor arrays and implications for chemotactic signaling. Elife 2014, 3, 1-16. [CrossRef] [PubMed]

28. Khursigara, C.M.; Wu, X.; Subramaniam, S. Chemoreceptors in Caulobacter crescentus: Trimers of receptor dimers in a partially ordered hexagonally packed array. J. Bacteriol. 2008, 190, 6805-6810. [CrossRef] [PubMed]

29. Tegunov, D.; Cramer, P. Real-time cryo-electron microscopy data preprocessing with Warp. Nat. Methods 2019, 16, 1146-1152. [CrossRef] [PubMed]

30. Lehtinen, J.; Munkberg, J.; Hasselgren, J.; Laine, S.; Karras, T.; Aittala, M.; Aila, T. Noise2Noise: Learning image restoration without clean data. In Proceedings of the 35th International Conference on Machine Learning, Stockholm Sweden, 10-15 July 2018.

31. Buchholz, T.-O.; Jordan, M.; Pigino, G.; Jug, F. Cryo-CARE: Content-aware image restoration for cryo-transmission electron microscopy data. In Proceedings of the 2019 IEEE 16th International Symposium on Biomedical Imaging (ISBI 2019), Venice, Italy, 8-11 April 2019.

32. Castaño-Díez, D.; Kudryashev, M.; Arheit, M.; Stahlberg, H. Dynamo: A flexible, user-friendly development tool for subtomogram averaging of cryo-EM data in high-performance computing environments. J. Struct. Biol. 2012, 178, 139-151. [CrossRef]

33. Mo, G.; Zhou, H.; Kawamura, T.; Dahlquist, F.W. Solution structure of a complex of the histidine autokinase chea with its substrate CheY. Biochemistry 2012, 51, 3786-3798. [CrossRef]

34. Webb, B.; Sali, A. Comparative protein structure modeling using MODELLER. Curr. Protoc. Bioinform. 2016, 54, 5.6.1-5.6.37. [CrossRef] [PubMed]

35. Humphrey, W.; Dalke, A.; Schulten, K. VMD: Visual molecular dynamics. J. Mol. Graph. 1996, 14, 33-38. [CrossRef]

36. Briegel, A.; Wong, M.L.; Hodges, H.L.; Oikonomou, C.M.; Piasta, K.N.; Harris, M.J.; Fowler, D.J.; Thompson, L.K.; Falke, J.J.; Kiessling, L.L.; et al. New insights into bacterial chemoreceptor array structure and assembly from electron cryotomography. Biochemistry 2014, 53, 1575-1585. [CrossRef] [PubMed]

37. Briegel, A.; Ames, P.; Gumbart, J.C.; Oikonomou, C.M.; Parkinson, J.S.; Jensen, G.J. The mobility of two kinase domains in the escherichia coli chemoreceptor array varies with signalling state. Mol. Microbiol. 2013, 89, 831-841. [CrossRef]

38. Fu, X.; Himes, B.A.; Ke, D.; Rice, W.J.; Ning, J.; Zhang, P. Controlled bacterial lysis for electron tomography of native cell membranes. Structure 2014, 22, 1875-1882. [CrossRef]

39. Wietrzynski, W.; Schaffer, M.; Tegunov, D.; Albert, S.; Kanazawa, A.; Plitzko, J.M.; Baumeister, W.; Engel, B.D. Charting the native architecture of Chlamydomonas thylakoid membranes with single-molecule precision. Elife 2020, 9, e53740. [CrossRef]

40. Natale, A.M.; Duplantis, J.L.; Piasta, K.N.; Falke, J.J. Structure, function, and on-off switching of a core unit contact between CheA kinase and $\mathrm{CheW}$ adaptor protein in the bacterial chemosensory array: A disulfide mapping and mutagenesis study. Biochemistry 2013, 52, 7753-7765. [CrossRef]

41. Piñas, G.E.; Frank, V.; Vaknin, A.; Parkinson, J.S. The source of high signal cooperativity in bacterial chemosensory arrays. Proc. Natl. Acad. Sci. USA 2016, 113, 3335-3340. [CrossRef]

42. Frank, V.; Piñas, G.E.; Cohen, H.; Parkinson, J.S.; Vaknin, A. Networked chemoreceptors benefit bacterial chemotaxis performance. mBio 2016, 7, e01824-16. [CrossRef]

43. Li, M.; Hazelbauer, G.L. Selective allosteric coupling in core chemotaxis signaling complexes. Proc. Natl. Acad. Sci. USA 2014, 111, 15940-15945. [CrossRef]

44. Keegstra, J.M.; Kamino, K.; Anquez, F.; Lazova, M.D.; Emonet, T.; Shimizu, T.S. Phenotypic diversity and temporal variability in a bacterial signaling network revealed by single-cell FRET. Elife 2017, 6, e27455. [CrossRef] [PubMed]

45. Muok, A.R.; Ortega, D.R.; Kurniyati, K.; Yang, W.; Maschmann, Z.A.; Mabrouk, A.S.; Li, C.; Crane, B.R.; Briegel, A. Atypical chemoreceptor arrays accommodate high membrane curvature. Nat. Commun. 2020, 11, 1-13. [CrossRef]

46. Erbse, A.H.; Falke, J.J. The core signaling proteins of bacterial chemotaxis assemble to form an ultrastable complex. Biochemistry 2009, 48, 6975-6987. [CrossRef] [PubMed]

47. Piasta, K.N.; Falke, J.J. Increasing and decreasing the ultrastability of bacterial chemotaxis core signaling complexes by modifying protein-protein contacts. Biochemistry 2014, 53, 5592-5600. [CrossRef]

48. Schulmeister, S.; Ruttorf, M.; Thiem, S.; Kentner, D.; Lebiedz, D.; Sourjik, V. Protein exchange dynamics at chemoreceptor clusters in Escherichia coli. Proc. Natl. Acad. Sci. USA 2008, 105, 6403-6408. [CrossRef] [PubMed]

49. Slivka, P.F.; Falke, J.J. Isolated bacterial chemosensory array possesses quasi- and ultrastable components: Functional links between array stability, cooperativity, and order. Biochemistry 2012, 51, 10218-10228. [CrossRef]

50. Sleytr, U.B.; Beveridge, T.J. Bacterial S-layers. Trends Microbiol. 1999, 7, 253-260. [CrossRef] 